\title{
Valoración integral del bosque de manglar en el municipio de San Andrés de Tumaco, Nariño, Colombia
}

\author{
Integrate assessment of mangrove forest in the Municipality of \\ San Andrés de Tumaco, Nariño, Colombia
}

\author{
Zulmary Valoyes Cardozo', Carlos Moreno Díaz², Catalina Sosa Botero ${ }^{3}$
}

\section{Resumen}

Teniendo en cuenta la importancia de los manglares del Pacífico colombiano, por su alta productividad y por su provisión de bienes y servicios, se estimó la disponibilidad a pagar de la población rural del municipio de Tumaco; para ello se realizaron 157 encuestas. La disponibilidad a pagar (DAP) fue calculada a través del método de valoración contingente. Los resultados de este estudio, permitieron afirmar que $99 \%$ de las personas encuestadas afirma conocer o haber recorrido el ecosistema de manglar y 92\% de los encuestados consideran que estos ecosistemas están amenazados; es por ello que $85 \%$ de los encuestados están de acuerdo en dar un aporte voluntario en dinero para conservar este ecosistema natural. Además de ello en caso de no aportar en dinero, los habitantes encuestados estarían dispuestos a dedicar unas horas de trabajo al mes o cambiar sus hábitos culturales en pro del mantenimiento de los servicios ecosistémicos que presta el ecosistema, por otro lado ni el sexo, ni la edad influyen en la disponibilidad a pagar para el mantenimiento de los manglares, pues la población en general entiende la importancia que tiene el ecosistema en cuanto a productividad y servicios de provisión.

Palabras clave: Disponibilidad a pagar, Manglares de Tumaco, Valoración económica.

\begin{abstract}
Having in it counts the importance of the swamps of the Colombian Pacifico, for his high productivity and for his provision of goods and services, the availability was estimated to paying of the rural population of Tumaco's municipality; for it 157 surveys were realized. The willingness to pay (WTP) was calculated across the method of valuation fix quotas. The results of this study, they allowed to affirm that $99 \%$ of the polled persons affirms to know or to have crossed the ecosystem of swamp, $92 \%$ of the polled ones thinks that these ecosystems are threatened; it is for it that $85 \%$ of the polled ones agrees in giving a voluntary contribution in money to preserve this natural ecosystem. Besides it in case of not reaching in money, the polled inhabitants would be ready to dedicate a few working hours a month or to change his cultural habits in favor of the maintenance of the services ecosistémicos that gives the ecosystem, on the other hand not even the sex, not even the age they influence the availability to paying for the maintenance of the swamps, since the population in general understands the importance that has the ecosystem as for productivity and services of provision.
\end{abstract}

1 Bióloga, Especialista en Administración de Recursos Naturales, Estudiante de Maestría en Ciencias Ambientales. Investigadora principal componente ecosistémico, Instituto de Investigaciones Ambientales del Pacífico(IIAP). Quibdó, Colombia.

e-mail: zvaloyes@iiap.org.co

2 Economista, Magister en Economía Agraria. Contratista Instituto de Investigaciones del Pacífico, Bogotá, Colombia. e-mail: carlosmorenodiaz@yahoo.com

3 Economista, Especialista en Gestión Ambiental, Estudiante de Maestría en Desarrollo Rural. Contratista Instituto de Investigaciones del Pacífico, Bogotá, Colombia.

e-mail: catasobot@gmail.com

Recibido: 23 de enero de 2014

Aprobado: 2 de abril de 2014 


\section{Bioetnia Volumen 11, 2014}

Keywords: Economic valuation, Tumaco's swamps, Willingness to pay.

\section{Introducción}

Los manglares son los humedales más conocidos por sus particularidades biológicas y su importancia económica; son comunidades halófitas y típicamente arbóreas, sujetas al efecto de las mareas. Por su carácter transicional en el ecotono tierra-agua, poseen características especiales que les permite adaptarse a un medio altamente dinámico (Windevoxhel 1992). Los manglares desde el punto de vista ecológico son muy productivos, representan comunidades vegetales muy importantes, ofrecen una variedad de hábitat a gran cantidad de especies de fauna terrestre y acuática de importancia ecológica y comercial, lo que les concede una especial importancia en términos de biodiversidad (Windevoxhel 1992, INVEMAR-CRC-CORPONARIÑO 2006).

Los manglares son ecosistemas que además del mangle incluyen los animales y plantas asociadas con un elemento forestal dominante y tolerante a la salinidad, crecen a lo largo del litoral en la zona entre mareas, en sitios protegidos con sedimentos lodosos y salinos. Las especies de mangles tienen la capacidad de adaptación a diferentes medios y condiciones ambientales, han desarrollado un sistema de raíces que les proporciona mayor estabilidad en condiciones de suelos blandos; poseen estructuras especializadas para el intercambio de gases a través de una serie de poros llamados lenticelas y los neumatóforos que son pequeñas raíces en forma de dedos que salen del suelo (Pizarro et al. 2004).

Desde la perspectiva social y económica, las zonas costeras han sido consideradas como parte de los ejes de desarrollo de los países, porque en ellas se establecen asentamientos humanos que hacen uso directo o indirecto de la oferta de recursos naturales en estas áreas, contribuyendo al establecimiento de diferentes tipos de actividades como pesca, acuicultura, industria, desarrollo de obras de infraestructura como vías y puertos, transporte marítimo y fluvial, agricultura, ganadería, turismo, comercio y minería entre otras (INVEMAR-CRC-CORPONARIÑO 2006).

Estos ecosistemas están dotados de una gran capacidad para proveer bienes y servicios que sostienen actividades económicas como la pesca, la acuicultura, el turismo, el transporte marítimo, la actividad portuaria, la explotación minera, las actividades agropecuarias y donde se dan asentamientos humanos e industriales importantes; sin embargo, la tendencia es desarrollar actividades que se justifican por su rentabilidad a corto plazo y por los beneficios que producen para sectores particulares, que por los beneficios que aportan en el largo plazo para la calidad de vida de la nación, lo que ha traído como consecuencia un crecimiento desordenado y una planificación deficiente así como problemas de contaminación, erosión de la línea de costa, degradación y pérdida de hábitat y disminución progresiva de los recursos pesqueros (INVEMAR-CRC-CORPONARIÑO 2006).

A pesar de los beneficios ofrecidos por estos ecosistemas, el recurso del manglar en la región ha sido objeto de actividades industriales como la producción de taninos, la extracción de postes, la extracción de leña y para la producción de carbón; aunque estas prácticas han correspondido a impulsos temporales, ha generado impactos de importancia, causando que en algunos sitios haya significado la pérdida de la estructura original de la vegetación y en el peor de los casos la erradicación total del bosque (Quiñones 2010).

Cuando un ecosistema natural existe simplemente y le provee con sus funciones ecológicas algunos beneficios sin ningún costo a la sociedad, lo único que puede expresar el valor económico de esos servicios ambientales es la "disposición a pagar" (DAP) que se tenga por conservar el ecosistema donde se generan, independientemente de si existe una erogación de tipo monetario o no. En esas circunstancias, ¿qué sentido tiene valorar los ecosistemas? La respuesta más clara es que si bien se sabe intuitivamente su importancia real, esto tal vez no sea suficiente para poder garantizar su aprovechamiento racional, por lo cual, apoyarse en los distintos instrumentos que las técnicas de valoración económica ambiental proporcionan es una interesante alternativa para ayudar a tomar las difíciles decisiones que estas situaciones exigen.

En términos generales, la valoración económica le apunta a que los recursos escasos se asignen de la mejor manera y a que se haga un uso eficiente de los mismos, porque su objetivo es contribuir a encontrar un nivel óptimo de bienestar social. Considerando este marco conceptual que suministra la economía ambiental, se realizó la valoración integral de los manglares de Tumaco, con el fin de obtener un valor económico que sirva de herramienta para la toma de decisiones en términos de manejo y conservación de este importante ecosistema natural.

\section{Área de estudio}

Localización y aspectos generales. San Andrés de Tumaco está ubicado en la costa pacífica nariñense, en cercanías de la frontera con Ecuador, entre las latitudes $1^{\circ} 45^{\prime}$ y $2^{\circ} 00^{\prime} \mathrm{N}$ y las longitudes $78^{\circ} 30^{\prime}$ y $78^{\circ} 45^{\prime} \mathrm{O}$. Se encuentra en la parte sur de la bahía de Tumaco, la cual tiene un área aproximada de $350 \mathrm{~km}^{2}$, con profundidades del agua que varían entre 0 y 50 metros (CCCP 2003).

Tumaco por estar ubicada entre la cordillera occidental y el océano Pacífico (Figura 1), presenta un régimen climático muy húmedo, con precipitaciones promedio de 3066.9 $\mathrm{mm}$ al año y humedad relativa de $86 \%$. La temperatura ambiente oscila entre $25.4^{\circ} \mathrm{C}$ en época seca y $26.1^{\circ} \mathrm{C}$ en 


\section{Valoración integral del bosque de manglar. Z Valoyes et al.}

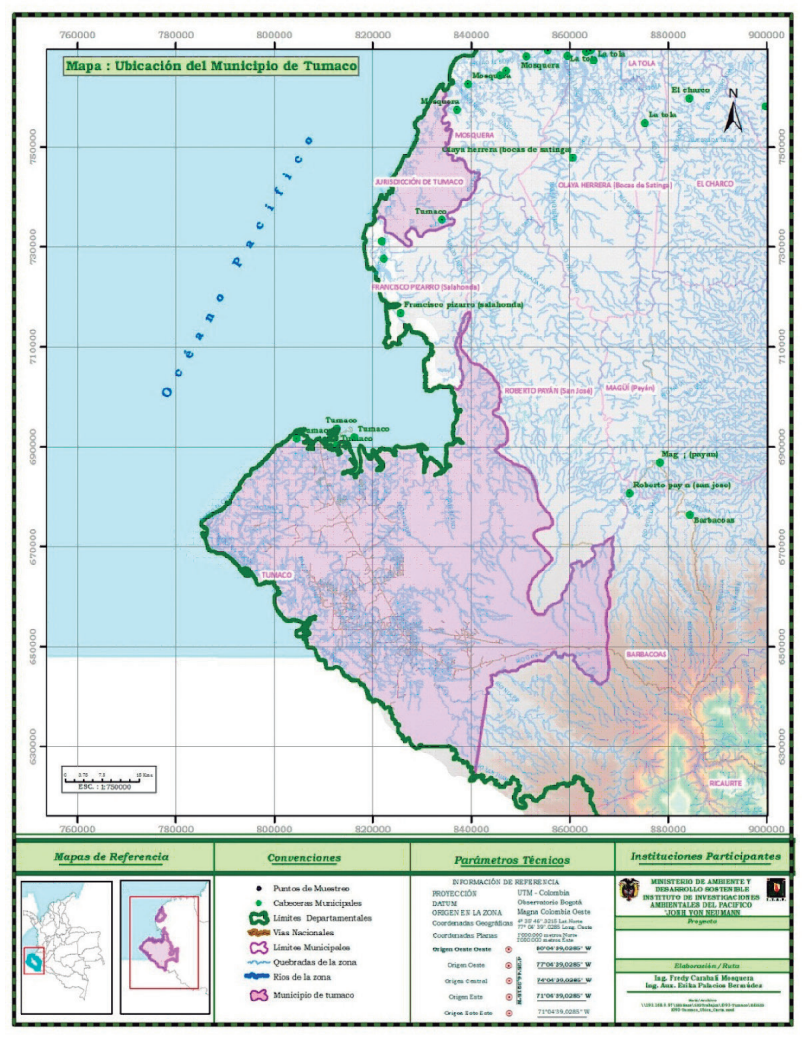

Figura 1. Ubicación de Tumaco.

época húmeda (CCCP 2003). Su casco urbano y zona de expansión están dispuestos en área continental e insular, esta última conformada por las islas de Tumaco, La Viciosa y El Morro, las cuales se encuentran unidas entre sí por los puentes El Pindo y El Morro. El municipio cuenta con más de 360 veredas en su zona rural, siendo el municipio de Colombia con mayor número de estas unidades territoriales.

\section{Aspectos socioculturales}

Población. Tumaco cuenta con una población total de 171.281 habitantes (año 2008) de los cuales 84.574 es población masculina (49.4\%) y 86.707 es población femenina (50.6\%) (Alcaldía Municipal 2010). Para el año 2003 según las proyecciones del DANE la población estimada para Tumaco era de 159.203 habitantes, de los cuales 80.108 ocupaban la cabecera urbana y el restante la zona rural. El mayor porcentaje poblacional se encuentra en las zonas de bajamar, con un promedio de 13.2 habitantes por kilómetro cuadrado. La gran mayoría del municipio está habitada por comunidades negras y en una pequeña proporción por comunidades indígenas (CCCP 2003).

Vivienda. La vivienda tradicional de los habitantes de Tumaco se levanta sobre pilotes de mangle, con paredes de machimbre, tablas de nato y techo de palma, aunque actualmente se observa una tendencia hacia las tejas de 'tejalit'. Se presenta una muy alta tasa de analfabetismo, con valores hasta del $20 \%$ en la zona urbana y $43.9 \%$ en el área rural. En el área urbana 60\% tiene primaria, 38\% secundaria y solo $2 \%$ ingresan a la educación superior. La recreación y el deporte se caracterizan por el mal estado de los escenarios deportivos (CCCP 2003).

Las condiciones de salud son muy deficientes, con una cobertura de 1.6 médicos por cada 10.000 habitantes, muy por debajo del pobre promedio nacional de 7 médicos. Existen algunos centros de salud de primer nivel y solo un centro de segundo nivel, cuya cobertura no alcanza a abarcar toda la población, por lo cual los casos graves se trasladan a Pasto o Cali.

Economía. De los 179.005 habitantes de Tumaco, 95\% de la población afrocolombiana es de raza negra. Sus formas de vida están íntimamente ligadas con el agua (mar, ríos, esteros, etc.) de donde se provee la mayor parte de recursos para el sustento diario. Basan su economía en actividades como la agricultura (sobre todo cultivo de coco y palma africana), la ganadería, minería, pesca, recolección de moluscos y crustáceos, cultivo de camarón, madera, quema de leña para extracción del carbón, carpintería, el comercio y la actividad del puerto. En el sector agrícola presentan productos como palma africana 14.800 ha, cacao 13.820 ha, plátano 3.900 ha, coco 3.408 ha; en el campo ganadero presenta 24.311 cabezas de ganado bovino, la explotación minera en los últimos 5 años aportó un promedio de 116,75 onzas de oro anuales (Alcaldía Municipal 2010). Tumaco es el segundo puerto marítimo del Pacífico colombiano, con una capacidad instalada de 5'276.729 toneladas. Su sistema portuario cuenta con 29 muelles, la gran mayoría no legalizados; 21 de ellos son pesqueros, tres generales, uno turístico, un amarradero y tres para venta de combustible; $51.7 \%$ de los muelles están construidos en madera y el resto en concreto (CCCP 2003).

Turismo. El turismo constituye una reserva potencial importante para Tumaco, con atractivos como esteros, mar y playas. Sus playas son el principal punto de atracción, por lo que allí se ubica la zona hotelera y de servicios turísticos, en especial sobre la playa de la Isla El Morro, al norte del área insular del municipio (CCCP 2003).

\section{Metodología}

Para conocer la percepción de la población de Tumaco en cuanto a la valoración del bosque de manglar, se realizaron 157 encuestas con preguntas semi-estructuradas. Las preguntas se elaboraron y se aplicaron no olvidando el aspecto cultural de la población y tomando en cuenta la relación entre el ser humano y el ecosistema de manglar.

Modelo matemático del método de valoración con- 


\section{Bioetnia Volumen 11, 2014}

tingente. El método de valoración contingente (MVC) plantea la construcción de un mercado para los bienes y/o servicios ecosistémicos que se quieren valorar, empleando como mecanismo el planteamiento de preguntas directas a los individuos "objetivo" sobre su disponibilidad a pagar, bajo escenarios o situaciones hipotéticas (Mendieta 2007, Carson 2000, Haab y McConnell 2002, Sarmiento y Prieto 2005, Riera et al. 2005). Este método por su construcción conceptual y matemática, es uno de los pocos que permite estimar una "proxy" del valor económico total de este tipo de bienes y/o servicios, porque recoge sus valores tanto de uso como de no uso (Mendieta 2007).

El MVC permite construir las preferencias de los individuos por el cambio en el bien y/o servicio que se está valorando (Mendieta 2005), lo cual implica que se debe tener buen conocimiento del punto de partida en términos de cantidad o calidad (línea base), así como del nivel en que puede situar a los individuos el escenario hipotético que haya sido propuesto. Esta información permite establecer la diferencia entre los beneficios obtenidos y los costos generados por preferirse una mejora del ecosistema analizado (Riera et al. 2005).

En términos generales, los pasos a seguir en un estudio de valoración contingente son:

- Definición del problema y determinación de una expresión analítica para determinar el cambio en el bienestar que puede ser trasladado a una pregunta o serie de preguntas.

- Formulación de la pregunta que revele la disponibilidad a pagar por el bien o servicio ambiental.

- Definición del grupo objetivo para la aplicación del cuestionario (estimación de la muestra).

- Determinación del método de muestreo (entrevista personal, telefónica o mediante correo).

- Realización de la encuesta piloto para detectary efectuar ajustes al cuestionario.

- Realización de muestreo completo al grupo objetivo.

- Realización de los análisis econométricos a las muestras (cuestionarios) recolectados.

El MVC, por medio del modelo de referéndum se basa en el enfoque de dar al entrevistado una elección y en el análisis de las elecciones hechas. Los entrevistados eligen la mejor alternativa, donde esta es la mejor medida de utilidad o la mayor disponibilidad a pagar, esto es una elección discreta. En lo referente a la estimación del modelo, el método denominado máxima verosimilitud es una alternativa a la estimación de mínimos cuadrados ordinarios (MCO), porque con este método, por medio de un proceso de iteraciones, se alcanzan unos estimadores insesgados asintóticamente.

Esta metodología tiene como principales objetivos los siguientes:

Evaluar principalmente los beneficios de proyectos que tienen que ver con bienes y/o servicios que no tienen un mercado definido.

- Estimar la disposición a pagar (DAP) o aceptar (DAA) como una proxy de la variación compensada (VC), o la variación equivalente (VE) respectivamente, con base en la percepción del beneficio o daño por parte del individuo encuestado.

Asimismo, los supuestos de esta metodología son:

- El individuo maximiza su utilidad dada una restricción de presupuesto representada por el ingreso disponible.

- El comportamiento del individuo en el mercado hipotético es equivalente a un mercado real.

- El individuo debe tener una completa información sobre los beneficios del bien, lo cual debe estar incluido en la pregunta de disponibilidad a pagar.

Modelo utilitario general. Hanemann(1984) desarrolló la formulación teórica del modelo de valoración contingente usando el formato de pregunta tipo referéndum, para estimar los cambios en el bienestar de las personas, formulando el problema como la comparación entre dos funciones indirectas de utilidad y formalizando el problema.

Supone que el entrevistado posee una función de utilidad $U(Q, Y ; S)$, que depende de la mejora de la calidad del bien ambiental $(Q)$ (estado actual $Q=0$ o final $Q=1$ ), del ingreso $(Y)$ y teniendo como parámetros el vector de características socioeconómicas $(S)$ del individuo. En razón a que el investigador desconoce la función $U(Q, Y ; S)$ de los entrevistados, entonces se plantea un modelo estocástico de la forma:

$$
U(Q, Y ; S)=V(Q, Y ; S)+\varepsilon(Q)
$$

Donde $\varepsilon(Q)$ es la variable aleatoria, independiente e idénticamente distribuida, con media cero y varianza constante y $V$ es la parte determinística de la función de utilidad. Se define la función de utilidad del individuo que responde No a la pregunta de disponibilidad a pagar como:

$$
V(0, Y ; S)+\varepsilon(0)
$$

Y la función de utilidad del individuo que responde Sí como $V(1, Y-P ; S)+\varepsilon(1)$. Cuando el entrevistado acepta pagar para disfrutar de la mejora en la calidad ambiental, debe cumplirse que:

$$
\begin{gathered}
V(1, Y-P ; S)+\varepsilon(1) \geq V(Q, Y ; S)+\varepsilon(0) \\
V(1, Y-P ; S)+V(Q, Y ; S)>\varepsilon(0)-\varepsilon(1) \\
N=\varepsilon(0)-\varepsilon(1)
\end{gathered}
$$

Donde $\varepsilon(0)$ y $\varepsilon(1)$ son dos variables aleatorias independientes e idénticamente distribuidas. Al simplificar lo anterior se tiene:

$$
\Delta V=V(1, Y-P ; S)-V(0, Y ; S)
$$




\section{Valoración integral del bosque de manglar. Z Valoyes et al.}

Y donde, $\eta=\varepsilon(0)-\varepsilon(1)$. Aquí la respuesta del entrevistado Sí/No es una variable aleatoria para el evaluador. La probabilidad de una respuesta Sí está dada por:

$$
\operatorname{Prob}(\text { decir Si })=\operatorname{Pr}(\Delta V>\eta)=F(\Delta V)
$$

Es decir que la probabilidad de que el individuo responda afirmativamente será igual a la probabilidad de que la utilidad con el cambio sea mayor a la utilidad en el estado inicial. Donde es la función de probabilidad acumulada de los errores representados por $\eta$.

Si suponemos una forma funcional para la función de utilidad lineal con respecto al ingreso (no existe efecto ingreso), se obtiene:

$$
V_{0}=\alpha_{0}+\beta Y+\varepsilon_{0}
$$

La función de utilidad del cambio estaría definida como:

$$
V_{1}=\alpha_{1}+\beta(Y-P)+\varepsilon_{1}
$$

Entonces la probabilidad de decir Sí al pago está dada por: $\operatorname{Prob}($ decir Sí $)=\operatorname{Prob}\left(\alpha_{1}+\beta(Y-P)+\varepsilon_{1}>\alpha_{0}+\beta Y+\varepsilon_{0}\right)$

$$
\begin{gathered}
\text { Si } \alpha=\alpha_{1}-\alpha_{0} \text { y } \eta=\varepsilon_{0}-\varepsilon_{1} \text { entonces: } \\
\operatorname{Prob}(\operatorname{decir} \text { Sí) }=\operatorname{Prob}(\alpha-\beta P>\eta)
\end{gathered}
$$

donde $\beta>0$, porque el valor esperado de la $V$ utilidad aumenta con el ingreso, implicando que cuanto más alto sea $P$ en la encuesta menor será $\Delta V$ y por tanto, menor será la probabilidad de que un individuo responda Sí.

Este método sólo permite estimar la diferencia $\alpha_{1}=\alpha_{1}$ $-\alpha_{0}$, representando el cambio de utilidad por la mejora de la calidad ambiental y $\beta$, representa la utilidad marginal del ingreso. Se verifica entonces que: $\mathrm{P}^{*}=\alpha / \beta$

$\mathrm{Si}$ a la función de probabilidad se le asocia una distribución normal para $\eta$, con media cero y varianza constante, es decir, $\eta \approx N\left(0, \sigma^{2}\right)$, se obtiene un modelo Probit, cuya probabilidad de respuesta Sí se modela como:

$$
\operatorname{Prob}(\text { decir Si })=\operatorname{Prob}(\alpha-\beta P) / \sigma>\eta / \sigma)=\int_{-\infty}^{\mu / \sigma} N(e)
$$

donde $e=\eta / \sigma$. Si los errores se distribuyen con un modelo Probit, la VC es:

$$
\mathrm{C}^{+}=\mathrm{DAP}=(\alpha / \sigma) /(\beta / \sigma)
$$

Si a la función de probabilidad se le asocia una distribución logística para $\eta$, se obtiene un modelo Logit, cuya probabilidad de respuesta Sí se presenta como:

$$
\operatorname{Prob}(\text { decir Si })=\operatorname{Prob}(\alpha-\beta P>\eta)=\left(1+\exp (-\alpha+\beta \mathrm{P})^{-1}\right.
$$

Si el investigador está interesado en encontrar la VC, que es la respuesta a la pregunta de DAP y los errores se distribuyen con un modelo logit, la $\mathrm{VC}$ es:

$$
C^{+}=D A P=\alpha / \beta
$$

La cual viene a ser la primera medida del bienestar, es decir, la media $C^{+}$de la distribución. Las magnitudes tanto para los modelos Probit como logit, son irrelevantes. Por ello los investigadores prefieren el modelo logit ya que admiten mayor varianza en la distribución del término de error. En un modelo de utilidad lineal como $V_{i}$ la media $C^{+}$ y la mediana $C^{*}$ son iguales. Si el investigador no permite valores negativos para $C$, entonces la medida monetaria del cambio en el bienestar dado a través de la media $C^{+}$ está dada por:

$$
C^{0=} C^{+}=\int^{\infty}(1-G c(\mathrm{P})) d \mathrm{P}=\log \left(1+\mathrm{e}^{\alpha}\right) / \beta
$$

Donde $G_{c}(\mathrm{P})$ da la probabilidad que $C$ sea menor o igual que $\mathrm{P}$, que es la probabilidad de obtener una respuesta negativa y $1-G c(\mathrm{P})$ da la probabilidad que $C$ sea mayor que P. Si se generaliza el procedimiento y se incluye el vector $S$, la medida del bienestar está dada por:

$$
C^{+=} C^{*}=D A P=\alpha_{0}{ }^{k} S / \beta=\left(\alpha_{0}+\sum_{i=1} \alpha_{i} S_{i}\right) / \beta
$$

Donde $S_{i}$ es el conjunto de características socioeconómicas, que no incluye el ingreso, $\alpha$ ' es la traspuesta del vector de parámetros y $\beta$ es el coeficiente del precio P. A continuación se presenta un resumen de la expresión matemática de las medidas de disponibilidad a pagar cuando se estima un modelo logit lineal y logit logarítmico.

Medidas de disponibilidad a pagar bajo el modelo logit lineal

$$
\begin{array}{cc}
\text { Media } & \text { Medina } \\
\mathrm{a} / \mathrm{b} & \mathrm{a} / \mathrm{b}
\end{array}
$$

Medidas de disponibilidad a pagar bajo el modelo logit logarítmico

$$
\begin{array}{cc}
\text { Media } & \text { Medina } \\
\operatorname{Exp}(-a / b) \cdot p /-b \cdot \operatorname{seno}(-p / b) & \operatorname{Exp~}\left(-a_{0} / b 1\right)
\end{array}
$$

Formatos de preguntas utilizados en estudios de valoración contingente. Las preguntas hipotéticas más utilizadas en valoración contingente tienen el objetivo de 


\section{Bioetnia Volumen 11, 2014}

averiguar el valor que le asignan las personas encuestadas a un cambio específico en un atributo ambiental, o la máxima disponibilidad que pueden tener las personas para acceder o conservar el bien analizado. Si las respuestas en realidad son verdaderas, representan expresiones directas del valor y por lo tanto deben ser interpretadas como una medida del excedente del consumidor. En conclusión, el término valoración contingente (VC) es convencionalmente empleado para referirnos a enfoques basados en esta forma de preguntas.

Un primer tipo de preguntas hipotéticas bajo este enfoque de valoración, está dirigido sólo a las que tienen un Sí o un No como respuesta. Un ejemplo sería, ¿Estaría usted dispuesto a pagar $\$ X$ cantidad de dinero por...? Cada una de las respuestas individuales revelan solamente un límite superior (para un No) o un límite inferior (para un Sí) de la medida de bienestar. Estas preguntas se han denominado preguntas de referéndum debido a su analogía con los procedimientos de entrevistas utilizados en estudios de votación electoral. Estos formatos de elección discreta pueden ser usados para estimar funciones de disponibilidad a pagar (DAP), o funciones de utilidad indirecta para datos que provengan de respuestas y características de los entrevistados.

Un segundo tipo de preguntas son las llamadas de ordenamiento contingente (ranking contingent). Alos entrevistados se les ofrece un conjunto de alternativas hipotéticas, cada una describiendo una situación distinta con respecto a algún atributo ambiental y otras características que se presumen son argumentos en la función de preferencias de los entrevistados. Los entrevistados ordenan las alternativas según sus preferencias. Este ranking puede ser analizado para determinar la tasa marginal de sustitución entre cualquier característica y el nivel del atributo ambiental. Si una de las otras características tiene un precio monetario, es posible estimar la disponibilidad a pagar de los entrevistados por el bien sobre la base del ordenamiento de las alternativas.

Una tercera clase de pregunta hipotética se conoce como formato de actividad contingente (activity contingent), en esta los individuos son interrogados sobre cómo debería cambiar el nivel de alguna actividad en respuesta a un cambio en el atributo ambiental. Por ejemplo ¿sí la contaminación en el agua de una playa se redujera, producto de un plan de saneamiento, en qué número incrementarían sus viajes por temporada? Sin embargo, si la actividad puede ser interpretada dentro del contexto de los modelos de comportamiento, tales como los modelos de conducta defensiva o por medio del modelo de costo de viaje para la estimación de la demanda por recreación, los métodos indirectos pueden ser más apropiados para obtener la disponibilidad a pagar de las personas por el bien, en lugar de utilizar el método de valoración contingente.
El principal aspecto a considerar en los métodos hipotéticos (valoración contingente) se refiere a la validez y el realismo de los datos, es decir, si las preguntas de naturaleza hipotética conducen o no a ciertas clases de sesgos o ruidos que hacen que la información conseguida con el encuestamiento no sirva para realizar inferencias válidas. Freeman (1979) identificó dos problemas esenciales de las preguntas hipotéticas:

Una tendencia de los entrevistados a comportarse estratégicamente, generando un sesgo en las respuestas, esperando influir sobre las decisiones que se puedan tomar con respecto al uso o conservación de algún ecosistema natural.

La ausencia de un incentivo en los entrevistados para proveer respuestas seguras cuando están siendo encuestados sobre situaciones hipotéticas.

Funcionamiento general del método de valoración contingente. Como se señaló antes, el método de valoración contingente tiene como propósito principal determinar el valor económico que le dan las personas al mantenimiento o al cambio en el bienestar generado por la conservación o la modificación de las condiciones en la oferta de un bien ambiental (uso). Esta valoración se obtiene a través de una pregunta directa. ¿Cómo es el funcionamiento del método? El punto de partida de este método son las encuestas, entrevistas o cuestionarios donde se debe tener en cuenta que la información sobre el objeto de estudio (ecosistema o servicio a ser valorado) debe ser la más relevante, y el entrevistado tiene que estar al tanto de esta información con el fin de tener pleno conocimiento del problema a tratarse. En el caso particular de los servicios ambientales es normal acompañar esta información con gráficos, fotografías o dibujos que ayuden a comprender el problema.

Otro aspecto importante a presentar, es el estado inicial y los beneficios que el mantenimiento o la modificación de las condiciones existentes del ecosistema o el servicio ambiental presenta para los individuos. Una vez descrito todo este escenario, lo siguiente es preguntar la disponibilidad a pagar por parte de las personas ante el status quo o el cambio propuesto, sin olvidar que cuando se trata de encontrar esta cantidad, el planteamiento que se haga debe girar siempre en torno al intercambio correspondiente a un bienestar igual o mayor por el dinero que se supone debe pagar a la persona encuestada. También es importante considerar en el cuestionario las características más relevantes de la persona entrevistada como la edad, estado civil, renta y nivel de estudios porque esta información adicional puede contribuir para determinar, sin ambigüedades, las preferencias de los individuos. En términos operativos, existen distintos formatos de pregunta entre las cuales se destacan los siguientes (Mendieta 2005):

Formato abierto. Aquí el entrevistador espera una respuesta a la pregunta que formula, esto tiene como desventaja 


\section{Valoración integral del bosque de manglar. Z Valoyes et al.}

que siempre se tiene un número alto de preguntas sin responder, por lo general atribuidas al poco conocimiento del entrevistado acerca de lo que podría ser una cifra razonable.

Formato subasta. Aquí se utiliza una variante y es que el entrevistador adelanta una cifra acerca de la disponibilidad a pagar del entrevistado, o si estaría dispuesto a pagar más por el ofrecimiento. Si la cifra es positiva, esta se eleva en una cantidad predeterminada y si es negativa se reduce hasta el punto donde el entrevistado plante su postura en una cifra.

Formato múltiple. Consiste en presentarle al entrevistado un cuadro en el que se le ofrecen distintas alternativas de cifras a pagar, ordenadas de mayor a menor y entre las cuales la persona debe seleccionar una.

Formato binario. Es conocido también como formato referéndum, aquí se le pregunta a la persona ¿pagaría usted tanto por...?¿Sí o No? Seleccionada una muestra representativa de la población, se subdivide en grupos igualmente representativos y se les hace la pregunta mencionada a cada uno de ellos con una cantidad diferente.

Formato iterativo. A partir de la pregunta inicial el entrevistador puede entrar en un juego iterativo con el entrevistado preguntándole por ejemplo "y si cambiamos... entonces ¿cambiaría usted su respuesta inicial? Esto permite que el entrevistado puede reflexionar con más cuidado acerca de la respuesta que está dando, el inconveniente es que dicho procedimiento permite dar respuestas más estratégicas que honestas.

\section{Valoración integral de ecosistemas de importancia ecológica y sociocultural en el Chocó Biogeográfico}

Se tomó en cuenta aspectos como el concepto de integralidad, para lo cual, se implementó desde el punto de vista técnico el método de valoración contingente (VC), porque a diferencia de los demás permite medir la disponibilidad a pagar como una proxy del valor económico total que tiene para un individuo conservar los beneficios que le presta un ecosistema natural debido a que recoge valores de no uso, valores de existencia, valores de opción y valores de legado. En términos operativos, esa medición se logra captando todos estos distintos valores a partir de las preferencias que expresen las personas a través de una encuesta estructurada para establecer la máxima disponibilidad a pagar por mantener la provisión actual o mejorar los servicios ambientales, económicos y culturales que les proporcionan los manglares objeto de estudio.

Desde una perspectiva general, esta investigación tuvo como principal objetivo valorar integralmente los manglares de Tumaco. A nivel más concreto, este trabajo investigativo permitió seleccionar un modelo logit para estimar la disponibilidad a pagar en el marco de la metodología de valoración contingente, porque su aplicación permite calcular la probabilidad asociada con el suceso $\mathrm{u}$ ocurrencia de un evento (paga, no paga), e identificar los distintos factores que inciden en su determinación. La interpretación matemática del modelo logit utilizado se puede hacer partiendo de la siguiente igualdad:

$$
Y_{i}=\frac{1}{1+\mathrm{e}^{-\mathrm{Zi}}}+\mu_{i}
$$

Teniendo a Y como una variable de respuesta binaria, y $\mu$ como una variable aleatoria que especifica los errores del modelo, al despejar Zi en la ecuación anterior se deduce que:

$$
Z_{i}=\int \boldsymbol{n} \frac{P_{i}}{1-P_{i}}
$$

Esta igualdad matemática muestra en el cociente la posibilidad de que se elija una alternativa frente a la otra opción contraria. En el caso concreto del modelo dicotómico, esta división planteada define en términos cuantitativos la probabilidad de que suceda un hecho o sea seleccionada la opción $1\left(\mathrm{P}_{i}\right)$, frente a la probabilidad de que no suceda el hecho o de que se seleccione la opción $0\left(1-\mathrm{P}_{i}\right)$, es decir contrapone la disponibilidad a pagar (DAP) por conservar un ecosistema y sus servicios ambientales con no hacerlo. El modelo logit efectúa comparaciones entre las diferentes elecciones de un mismo individuo, las cuales a su vez están determinadas por un conjunto de regresores. En la comparación de las elecciones, la afectación del bienestar se calcula equiparando el valor de la variable cuando es igual a uno (1), con la utilidad del individuo cuando el valor de la variable es cero (0), proporcionando el siguiente resultado:

$$
\frac{\mathrm{P}_{i} \mid X_{2 i}=1}{\operatorname{1-P}_{i} \mid X_{2 i}=0}=e^{\beta 2}
$$

Como es evidente, la relación probabilística expresada en la anterior igualdad matemática permite en términos microeconómicos establecer que las personas toman sus decisiones utilizando por regla general parámetros constantes. En este contexto, la conservación de los ecosistemas y sus distintos servicios ambientales dependerá de la DAP que expresen los individuos por un aumento en el nivel de bienestar. Estas decisiones discretas no admiten cambios en las cantidades consumidas, por lo tanto, la elección que 


\section{Bioetnia Volumen 11, 2014}

haga un individuo puede representarse como un problema de maximización de la función de utilidad sujeta a una restricción presupuestaria, lo cual puede ser establecido en un modelo matemático categórico (logit) que incluya variables binarias.

Considerando lo anterior y ajustando a Hanemann(1984) se puede establecer que las principales formas funcionales que permiten medir el cambio en la utilidad de una persona derivado del cambio en la conservación de los ecosistemas y sus servicios ambientales son:

Lineal en función del valor de la DAP:

$\Delta V=\boldsymbol{\alpha}_{0}-\beta_{1} v d a p$

Lineal en función del valor de la DAP y del ingreso:

$\Delta V=\boldsymbol{\alpha}_{0}-\beta_{1} v$ dap $+\beta_{2}$ ingreso

Lineal en función del valor de la DAP, del ingreso y de variables socio demográficas:

$\Delta V=\boldsymbol{\alpha}_{0}-\beta_{1} v$ dap $+\beta_{2}$ ingreso $+\ldots .+\beta_{n} v s_{n}$

Lin - Log en función del valor de la DAP:

$\Delta V=\boldsymbol{\alpha}_{0}-\beta_{1} \log$ (vdap)

Lin - Log en función del valor de la DAP y del ingreso:

$\Delta V=\boldsymbol{\alpha}_{0}-\beta_{1} \log ($ vdap $)+\beta_{2} \log$ (ingreso)

Lin - Log en función del valor de la DAP, del ingreso y de variables socio demográficas:

$\Delta V=\boldsymbol{\alpha}_{0}-\beta_{1} \log ($ vdap $)+\beta_{2} \log ($ ingreso $)+\ldots .+\beta_{n}\left(v s_{n}\right)$

Todas estas formas funcionales pueden ser estimadas con modelos logit para encontrar la máxima disposición a pagar (DAPmáx); sin embargo, por la estructura homogénea de las encuestas diseñadas y la calidad de los datos obtenidos durante su implementación se optó por utilizar la forma "lineal en función del valor de la DAP, del ingreso y de variables sociodemográficas" con el objeto de obtener un valor económico que ayude a tomar decisiones en términos de manejo y conservación de cinco importantes ecosistemas naturales del Chocó Biogeográfico, como se muestra a continuación.

La base matemática estará sustentada por la formulación de Hanemann (1984), la cual se operativiza a partir de la información recolectada mediante formatos de preguntas tipo referéndum, para estimar los cambios en el bienestar de las personas planteando el problema a ser analizado como la comparación entre dos funciones indirectas de utilidad y formalizando dicho problema de la forma como se describe a continuación:

Supone que el entrevistado posee una función de utilidad $\mathrm{U}(\mathrm{Q}, \mathrm{Y} ; \mathrm{S})$, que depende de la mejora de la calidad del bien o servicio ambiental (Q) (estado actual $Q=0$ o final $Q=1$ ), del ingreso $(\mathrm{Y})$ y teniendo como parámetros el vector de características socioeconómicas (S) del individuo. Como el investigador desconoce la función U $(\mathrm{Q}, \mathrm{Y} ; \mathrm{S})$ de los entrevistados, entonces se plantea un modelo estocástico de la forma:

$$
\mathrm{U}(\mathrm{Q}, \mathrm{Y} ; \mathrm{S})=\mathrm{V}(\mathrm{Q}, \mathrm{Y} ; \mathrm{S})+\varepsilon(\mathrm{Q})
$$

Donde $\varepsilon(\mathrm{Q})$ es la variable aleatoria, independiente o idénticamente distribuida, con media cero y varianza constante y $\mathrm{V}$ es la parte determinística de la función de utilidad. Si se define la función de utilidad del individuo que responde No a la pregunta de disponibilidad a pagar como:

$$
\mathrm{V}(0, \mathrm{Y} ; \mathrm{S})+\varepsilon(0)
$$

y la función de utilidad del individuo que responde Sí como:

$$
\mathrm{V}(1, \mathrm{Y}-\mathrm{P} ; \mathrm{S})+\varepsilon(1)
$$

Si el entrevistado acepta pagar para disfrutar de la mejora en la calidad ambiental, debe cumplirse que:

$$
\mathrm{V}(1, \mathrm{Y}-\mathrm{P} ; \mathrm{S})+\varepsilon(1) \geq \mathrm{V}(\mathrm{Q}, \mathrm{Y} ; \mathrm{S})+\varepsilon(0)
$$

\section{Formulación para establecer el tamaño muestral}

Para establecer el tamaño muestral, se determinó el número de encuestas realizadas aplicando la siguiente formulación estadística:

$$
\left.\mathrm{n}=\left[\left(\mathrm{k}^{\wedge} 2 \times \mathrm{p} \times \mathrm{q} \times \mathrm{N}\right)\right) /\left(\mathrm{e}^{\wedge} 2 \times(\mathrm{N}-1)+\mathrm{k}^{\wedge} 2 \times \mathrm{p} \times \mathrm{q}\right)\right]
$$

Dónde:

$\mathrm{n}=$ Es el tamaño de la muestra

$\mathrm{p}=$ Probabilidad de éxito

$\mathrm{q}=$ Probabilidad de fracaso $(1-\mathrm{p})$

$\mathrm{N}=$ Es el tamaño de la población o universo (representa el número total de posibles personas encuestadas).

$\mathrm{k}=$ Es una constante que depende del nivel de confianza que asignemos. El nivel de probabilidad de que los resultados de la investigación sean ciertos. Por ejemplo, 95\% de confianza es equivalente a decir que nos podemos equivocar con una probabilidad del 4,5\%.

Los valores $\mathrm{k}$ más utilizados y sus niveles de confianza ( $\%)$ : 1,15 (75) 1,28 (80) 1,44 (85) 1,65 (90) 1,96 (95) $2(95,5)$ 2,58 (99)

$\mathrm{e}=\mathrm{El}$ error muestral es la diferencia que puede haber entre el resultado que obtenemos preguntando a una muestra de la población y el que obtendríamos si preguntáramos al total de ella.

\section{Resultados}

Como se señaló antes, la estimación de la muestra dio un total de 378 encuestas de las cuales se aplicaron 157 en la zona rural del municipio de Tumaco, cuyos resultados se analizarán a continuación siguiendo los módulos temáticos 


\section{Valoración integral del bosque de manglar. Z Valoyes et al.}

en que fue estructurada la encuesta.

$\mathrm{Al}$ indagar sobre la familiaridad que tienen las personas encuestadas con los manglares de Tumaco se encontró que $99 \%$ han recorrido por lo menos una vez este ecosistema, lo cual significa que la mayoría de los encuestados dan su opinión con elementos de juicio soportados en el conocimiento previo y directo de la zona. De esta manera, cuando se preguntó si creían que desde el punto de vista ambiental estos manglares se encuentran amenazados, $92 \%$ respondieron afirmativamente y piensan que la principal amenaza que presenta el ecosistema se debe sobre todo a la tala de árboles, mientras que $8 \%$ expresó no percibir ninguna amenaza, lo cual posiblemente pueda estar relacionado con las acciones que se han emprendido en el departamento por parte de la Corporación Autónoma y los consejos comunitarios en pro de la protección del ecosistema, lo que permite que cierta parte de la población considere que al emprender medidas de protección esto hace que el ecosistema presente menor riesgo. La afirmación a creer que los manglares de Tumaco se encuentran amenazados puede tener una relación directa con el hecho que casi todas las personas encuestadas viven dentro de los manglares y se benefician directamente de estos, lo que ha permitido que los pobladores sean conscientes del deterioro a que han sido sometidos estos ecosistemas por diferentes actividades antrópicas.

Por otra parte, al ser el recurso hídrico uno de los bienes ambientales que diariamente utiliza y se beneficia la población, se indagó por la fuente que los abastece y la calidad de la misma. El 99\% de las personas encuestadas respondieron que su fuente de agua principal es por medio de pozos y la colectación de agua lluvia, al no contar ninguno con sistema de acueducto en sus hogares. Por consiguiente, en esta zona de estudio aún las personas se suplen del recurso vital agua por medio de mecanismos precarios que no les garantiza calidad ni continuidad del mismo. Lo anterior se debe a que la población encuestada vive en el área rural lo que impide que cuenten con servicios de agua potable y alcantarillado, condición que se cumple en casi toda la costa pacífica donde la población rural no cuenta con los principales servicios y la población más beneficiada es la que vive en el área urbana.

Así, $42 \%$ de los encuestados considera que la calidad del agua que toman en sus hogares no es buena, porque asocian distintas enfermedades que han padecido familiares y personas de la zona por el consumo mismo de esa agua con que se abastecen. Y por otra parte, $99 \%$ manifiesta que la disponibilidad de dicho recurso es intermitente durante todo el año y su permanencia está sujeta a las condiciones climáticas de la zona y los recursos económicos de los hogares.

$\mathrm{Al}$ momento de preguntarle a las personas el tipo de trabajo al cual se dedican y por el cual perciben ingresos para sostener sus hogares, se encontró que la agricultura ocupa el primer lugar con $64 \%$, seguida de la pesca $(14 \%)$ $\mathrm{y}$ otros trabajos diferentes a las actividades de campo (9\%) destacándose el comercio y oficios varios. Del total de encuestados 95\% expresa que su trabajo lo realiza en zona de los manglares de Tumaco, siendo la agricultura la que mayor presencia tiene $(66 \%)$ seguida por la pesca $(15 \%)$.

Identificadas las principales actividades productivas, se les preguntó a las personas por los ingresos y gastos mensuales que tienen sus hogares, con el objeto de tener indicios del nivel socioeconómico de la zona de estudio. Así, con los datos recolectados y aplicando el método de promedio ponderado, se estimó que un hogar de cuatro personas en promedio tiene unos ingresos mensuales de $\$ 474,255$ pesos y gastos de $\$ 426,859$, lo que en términos económicos significaría un balance positivo en las finanzas del hogar representado por un excedente aproximado del $10 \%$. Los anteriores resultados están relacionados con el tipo de actividad económica de la población objeto de estudio la cual está basada sobre todo en la agricultura (cultivo de palma africana, cacao y coco) y el tipo de trabajo ejercido por los encuestados quienes en su mayoría son jornaleros de fincas agrícolas cuyo salario no supera los dos salarios mínimos mensuales.

A partir de un estudio realizado por el equipo de profesionales del proyecto, se lograron identificar y definir los principales servicios ambientales que prestan los manglares de Tumaco, con el propósito de dárselos a conocer a las personas encuestadas para que expresaran su conocimiento al respecto y la importancia que estos le merecen. Entre los doce servicios ambientales presentados en la encuesta, las personas reconocieron especialmente tres de estos: a) protección contra inundaciones y tormentas; $b$ ) conservación de flora y fauna; c) conservación y estabilización de la línea costera. En relación con el nivel de importancia que tienen estos servicios para las personas, se obtuvo como respuesta que todos fueron valorados entre importantes y muy importantes porque se considera que los mismos son imprescindibles para mejorar la calidad de vida de las personas y que sin ellos se puede perder el legado cultural que para las mismas representan los manglares de Tumaco.

La valoración económica propuesta y desarrollada en el presente estudio se basó en el método de valoración contingente, que a través de una pregunta directa de DAP busca conocer si la persona encuestada estaría o no dispuesta a dar voluntariamente un aporte monetario para la conservación de los manglares de Tumaco. De esta manera, se obtuvo como resultado que $85 \%$ de los encuestados respondieron afirmativamente a dar un aporte voluntario en dinero para conservar este ecosistema natural y $15 \%$ rechazó esta medida. Entre los motivos que tuvieron las personas para no estar dispuestas a pagar, se encuentra 


\section{Bioetnia Volumen 11, 2014}

principalmente la escasez de recursos económicos (61\%) y el no considerar necesario hacer este tipo de aportes para conservar el ecosistema (26\%). La disponibilidad a dar un aporte voluntario por los servicios prestados por el ecosistema puede estar directamente relacionada con la cantidad de dinero que ganan las personas encuestadas y los oficios que desempeñan, algunas de las personas son amas de casa, las cuales no devengan un salario y por lo tanto la disponibilidad a pagar se reduce.

Considerando el grupo de personas encuestadas que respondieron afirmativamente la pregunta de DAP, a partir de los cinco rangos de pagos propuestos en la encuesta se tiene que $57 \%$ estuvo de acuerdo en aportar menos de $\$ 20.000$ pesos al año, $35 \%$ entre $\$ 20.000-\$ 30.000$, $4 \%$ entre $\$ 30.001-\$ 40.000,1 \%$ entre $\$ 40.001-\$ 50.000,1 \%$ más de $\$ 50.000$ y el $1 \%$ restante no eligió alguna alternativa propuesta.

Con el objetivo de conocer el interés de las personas por coadyuvar a conservar los manglares de Tumaco más allá de estar o no dispuestos a dar un aporte monetario, se les preguntó también si apoyarían esa iniciativa dedicando horas de trabajo físico en los manglares, o con cambios en el aprovechamiento productivo de los recursos naturales que provee este ecosistema $\mathrm{y} / \mathrm{o}$ con cambios culturales en el hogar que ayuden a disminuir la contaminación del entorno. Frente a lo cual $64 \%$ de las personas encuestadas expresaron estar dispuestas a dedicar horas de trabajo para este propósito de mejoramiento y conservación ambiental del ecosistema.

En cuanto a las características sociodemográficas de las personas encuestadas, se tiene que $75 \%$ fueron hombres y $25 \%$ mujeres, entre los cuales no hay gran diferencia de quién presenta mayor disponibilidad a pagar, porque alrededor $84 \%$ tanto de hombres como de las mujeres encuestados respondieron afirmativamente a la DAP.

Con respecto a la edad, la encuesta se aplicó de manera muy heterogénea porque se encuestaron personas entre los 18 y 85 años, lo cual permitió tener un mayor margen de exploración respecto a la relación entre edad, percepción frente a los problemas ambientales que soporta el ecosistema y la disponibilidad a pagar para apoyar su conservación. Analizando en intervalos de quince años la edad de la población encuestada, se tiene que el grupo más representativo es aquel entre los 18 y 33 años (33\%), seguido por los de 34 y 49 años (27\%), los de 50 y 65 años (26\%) y por último el de mayor edad, que varía entre los 66 y 85 años (13\%).

En relación con el nivel educativo, se tiene que $83 \%$ de las personas que respondieron a esta pregunta cursaron primaria, $14 \%$ secundaria, $2 \%$ cuentan con algún grado tecnológico y $1 \%$ son universitarios. Haciendo un análisis primario de la relación que existe entre el nivel educacional y la DAP de las personas, se observa que en todos los niveles de educación la DAP se registra entre $80 \%$ y $95 \%$, lo que muestra que en la zona de estudio la disposición de pago de las personas se da por igual en todos los niveles educativos.

\section{Disponibilidad a pagar (DAP) de las personas para conservar el ecosistema manglares de Tumaco}

La máxima disponibilidad a pagar de las personas encuestadas, se encuentra en un rango muy conservador cuyo valor mínimo es de $\$ 5.000$ pesos anuales y el valor máximo escasamente llega a los $\$ 50.000$ pesos anuales. Si se analizan por cuartiles ${ }^{1}$ los valores hipotéticos máximos a pagar, se tendría el siguiente panorama: $21 \%$ de los encuestados que respondieron Sí a la pregunta de DAP, registran un pago máximo entre $\$ 5.000$ y $\$ 9.999$ (primer cuartil); $27 \%$ entre $\$ 10.000$ y $\$ 19.999$ (segundo cuartil); $45 \%$ entre $\$ 20.000$ y $\$ 29.999$ (tercer cuartil); y $7 \%$ entre $\$ 30.000$ y $\$ 50.000$ (cuarto cuartil). Por lo tanto, se puede observar que las respuestas afirmativas a la pregunta de DAP para conservar los manglares de Tumaco no estuvieron concentradas mayoritariamente en el monto mínimo (ㅁ? 10.000 pesos), sino que al contrario, $52 \%$ se inclinó por seleccionar pagos entre $\$ 20.000$ y $\$ 50.000$ pesos.

Del mismo modo, la variable ingreso en este modelo logit es significativa como variable explicativa $(\mathrm{p}>|\mathrm{Z}|=0.195)$ a un nivel de confianza del $10 \%$. Su relación con la DAP (rdap) es positiva, lo cual coincide con la teoría económica e indica que los individuos al tener mayores ingresos muestran una probabilidad más alta de responder afirmativamente (Sí) a la pregunta de DAP para la conservación de los manglares de Tumaco.

Con respecto a la variable socioeconómica considerada en el modelo, se tiene que la variable educación es poco significativa a un nivel de confianza del $5 \%(\mathrm{P}>|\mathrm{Z}|=0.377)$, pero asimismo, el signo negativo de su coeficiente permite ver que en este caso no se cumple la relación esperada entre esta variable y la DAP, porque se supone que a mayor nivel educativo de la persona más alta es la probabilidad de que responda afirmativamente a la DAP, situación que se debe entender a partir de la información que aportaron las encuestas sobre el contexto educativo de la población, donde se observa que $83 \%$ de las personas encuestada cuentan con primaria y $17 \%$ restante con secundaria, tecnológica y universitaria; por consiguiente, aun cuando $73 \%$ de las personas con educación secundaria, tecnológica y universitaria expresaron una respuesta afirmativa a la DAP, de igual manera lo expresó $81 \%$ de las personas con nivel

1 Los cuartiles dividen la distribución de los datos analizados en cuatro partes, correspondientes a $0.25,0.50$ y 0.75 


\section{Valoración integral del bosque de manglar. Z Valoyes et al.}

de primaria, lo cual tuvo mayor representatividad en los resultados que arrojó el modelo con respecto a la relación entre la variable educación y la variable dependiente rdap.

Estimación de la DAP. El valor promedio que los habitantes de la zona rural del municipio de Tumaco, estarían dispuestos a pagar para conservar los servicios ambientales, económicos y culturales que les proporciona los manglares de Tumaco es de $\$ 6.071$ pesos por persona al año (Tabla 1), lo cual representa el valor que los ciudadanos pagarían voluntariamente para continuar disfrutando los servicios que les presta sin ningún costo ${ }^{2}$ este ecosistema natural (Tabla2).

La estimación de la máxima disponibilidad a pagar de las personas se considera como un indicador de la importancia que tiene para la población de la zona conservar los manglares de Tumaco, al estar dispuestos a desprenderse de un porcentaje de su ingreso con el propósito de contribuir a la protección del mismo. En este sentido, el ejercicio de análisis cualitativo y cuantitativo de la percepción de las personas por conservar los manglares de Tumaco, son una base instrumental para la toma de decisiones dirigidas a la atenuación de actividades que generan impactos negativos sobre el ecosistema y a la vinculación de las personas en el proceso integral de mantener y mejorar las condiciones de este ecosistem

\section{Conclusiones}

La aplicación del método de valoración contingente, permitió conocer los beneficios que las comunidades de Tumaco perciben o atribuyen a contar con un ecosistema de vital importancia como los manglares y los costos que cualquier nivel de intervención implicaría en la disponibilidad de los bienes y servicios ambientales. En este sentido los cálculos mostraron una clara disponibilidad a pagar por usar y conservar no solo los recursos naturales, sino también los aspectos culturales derivados de la existencia de ese ecosistema.

Se pudo analizar que los habitantes del área rural del municipio de Tumaco consideran que los manglares son un ecosistema de vital importancia para ellos, debido a que estos no solo les proporcionan bienestar, además de bienes o los recursos necesarios para vivir, razón por la cual están dispuestos a pagar para que el ecosistema se mantenga y les siga proveyendo los beneficios que hasta la actualidad reciben. Aunque solo 1\% esté dispuesto a pagar más de $\$ 50.000$ y la gran mayoría (57\%) manifestaron que solo tenían disponibilidad para pagar menos de $\$ 20.000$ no hace menos importante el ecosistema porque los ingresos que obtiene la población encuestada no permiten que haya mayor

2 Carecen de costo porque no tienen un mercado donde se puedan transar. disponibilidad a pagar; es importante destacar que $85 \%$ de la población está dispuesta a pagar para que el ecosistema se mantenga. Por otro lado, el nivel de educación de las personas juega un papel primordial al momento de preguntarles por su disponibilidad de pago hipotético para la conservación y restauración del ecosistema; $83 \%$ de la población encuestada solo han cursado hasta el nivel de primaria, lo que impacta en la relación que puedan establecer las personas entre la conservación del ecosistema y su bienestar a largo plazo al no asociar los bienes y servicios ambientales de los cuales se benefician y obtienen gracias al manglar.

Otro aspecto a considerar en el resultado obtenido de DAP está relacionado con la actividad económica que realizan las personas encuestadas; $64 \%$ se dedica a la agricultura, actividad que no se realiza dentro del manglar, mientras que actividades como la pesca y la extracción forestal que están directamente relacionada con los manglares son desarrolladas solo por $14 \%$ y $2 \%$ de la población encuestada, es decir que la mayoría de la población no tiene una conexión diaria productiva con el ecosistema por lo cual se puede ver influida en su percepción de importancia en conservar y restaurar ese ecosistema. Con respecto a la manifestación de rechazo a una DAP, se tiene que aunque $15 \%$ de la población encuestada que por razones económicas o por desconfianza al manejo de los recursos no estarían dispuesta a dar un aporte en dinero, este mismo grupo está dispuesto a dar su aporte en horas de trabajo y de esta forma contribuir al mejoramiento y conservación ambiental del ecosistema.

De esta manera, es posible concluir que la valoración económica realizada para este ecosistema se constituye en un ejercicio de aproximación al diseño de herramientas que puedan funcionar como incentivos de conservación, permitiendo no solo mantener la estabilidad de este tipo de ecosistemas, sino también la subsistencia, el mejoramiento de la calidad de vida de las comunidades que los han conservado a través de los años y la preservación de las tradiciones culturales que se desarrollan a su alrededor y que tienen un valor incalculable para quienes lo han habitado y mantenido en buenas condiciones ambientales.

\section{Literatura citada}

Alcaldía Municipal de Tumaco. 2010. Caracterización de San Andrés de Tumaco. Disponible en: www.tumaco-narino.gov.co/apc-aa.../CARACTERIZACION_2010.p.

Carson RT. 2000. Contingent valuation: A user's guide, "Environmental". Sci Fenol. 34 (8): 1413-8.

Centro de Control de Contaminación del Pacífico (CCCP). 2003. Aportes al entendimiento de la bahía de Tumaco. Entorno oceanográfico, costero y de riesgos. Serie de Publicaciones Especiales. 2: 183.

Freeman AM. 1979. Hedonic prices, property values and measuring environmental benefits: A survey of the issues. Scand J Econ. 81 (2): 154-73.

Haab TC, Mcconnell KE.. 2002. Valuing environmental and natural resources: The econometrics of non-market valuation (New Horizons in Environ- 


\section{Bioetnia Volumen 11, 2014}

mental Economics). Northampton: Edward Elgar Publishers.

Hanemann MW. 1984. Welfare evaluations in contingent valuation experiments with discrete responses. Am J Agricult Econ. 66: 322-41.

INVEMAR, CRC, CORPONARIÑO. 2006. Unidad Ambiental Costera de la Llanura Aluvial del Sur: Caracterización, diagnóstico integrado y zonificación ambiental. López A (ed.). Santa Marta: INVEMAR, CRC, CORPONARIÑO; 383 pp + cartografía anexa.

Mendieta J. 2005. Manual de valoración económica de bienes no mercadeables. Bogotá: CEDE, Universidad de los Andes.

Mendieta J. 2007. Herramientas microeconómicas básicas para el estudio de las metodologías de valoración ambiental y su aplicabilidad práctica en la evaluación económica de políticas y proyectos ambientales. Bogotá: CEDE, Universidad de los Andes.
Pizarro F, Piedra L, Bravo J, Asch J, Asch C, Bernal F, et al. 2004. Manual de procedimientos para el manejo de los manglares Costa Rica. Heredia: EFUNA; 132 pp.

Quiñones JJ. 2010. Los manglares en el municipio de Tumaco (Nariño). Pasto: Universidad de Nariño, Facultad de Educación.

Riera P, García D, Kriström B, Brännlund R. 2005. Manual de economía ambiental de los recursos naturales.

Sarmiento MA, Prieto RA. 2005. Métodos de valoración ambiental: un nuevo método basado en la variación del producto interior bruto. Madrid: Dirección General del Catastro; pp. 59-91.

Windevoxhel LNJ. 1992. Valoración económica parcial de los manglares de la región II de Nicaragua. Turrialba: Centro Agronómico Tropical de Investigación y Enseñanza. 